\section{Diagnostic Imaging for Pharmacists}

Smith BT, Weatherman KD, editors. American Pharmacists Association, Washington, DC, 2012. 273 pages, softcover. ISBN 978-1-58212-153-6. US\$74.95 (\$60 for APhA members).

Diagnostic Imaging for Pharmacists is a combined textbook and partial pharmacopeia that provides explanations of both diagnostic and therapeutic radiographic modalities and discussions of applicable contrast media, adjuncts, and therapeutic agents. It offers basic descriptions of the principles of imaging used in various techniques and delves more specifically into the associated pharmaceuticals necessary for imaging and therapy.

This text is, according to the website of the American Pharmacists Association, "the only diagnostic imaging reference written specifically for pharmacists." With its focus predominantly on the pharmaceuticals used within the radiology department, the most likely target audience would be pharmacists with responsibilities for the provision of care in this setting, an evolving role that the authors of this text advocate. Other pharmacists with an interest in the topic and those who regularly manage care for patients undergoing radiological procedures may also find this text useful.

The lead editors for this text are both pharmacists with interests in nuclear medicine. Smith holds a BScPharm and a $\mathrm{PhD}$ and is past chair of the University of Saint Joseph School of Pharmacy in West Hartford, Connecticut. Weatherman holds the credentials PharmD, BCNP, and FAPhA. She is a clinical associate professor of pharmacy practice at Purdue University in Indianapolis, Indiana, with primary responsibilities in nuclear pharmacy and diagnostic imaging. The 10 contributing authors are a group of physicians, pharmacists, radiation safety officers, and technical experts from various academic, industry, and medical institutions across the United States. This collection of authors has the broad scope and knowledge base necessary to produce a text such as this.
The book is divided into 13 chapters, with the first covering the role of the pharmacist in diagnostic imaging and focusing on the process of medication therapy management. The second chapter provides an introduction to the physics of ionizing radiation. The next 10 chapters review the imaging procedures, adjunct agents, and radiopharmaceuticals used in radiography, computed tomography, nuclear medicine, positron emission tomography, magnetic resonance imaging, and ultrasonography. The final chapter reviews regulations in the United States associated with the use of ionizing radiation.

These chapters provide adequate detail for understanding how the various imaging techniques function, with useful information on dosing, administration, contraindications, adverse effects, and medication therapy management issues for the various agents applicable to each imaging modality.

Overall, this book provides a solid foundation for pharmacists who want to improve their understanding of medical imaging, with sufficient detail about the associated pharmaceuticals to facilitate better monitoring of their effects. The only major limitation, in my opinion, is the lack of a section on the application of medical imaging in assessing other drug therapies. Appreciating that many drugs have beneficial and adverse effects that can be monitored with imaging and that most pharmacists have limited training in the use of these tools, such an addition would greatly expand the potential audience for the book. That said, information on the application of medical imaging for pharmacists would likely require a whole separate volume, given the potential amount of information to be covered.

Jerrold L Perrott, BScPharm, ACPR, PharmD

Pharmacy

Royal Columbian Hospital

New Westminster, British Columbia 